\title{
Correction to: Platelet-derived growth factor-BB induces pulmonary venous smooth muscle cells proliferation by upregulating calcium sensing receptor under hypoxic conditions
}

\author{
Rongmin Liu • Juan Xu Y Yongliang Jiang - Wei Hong • Shaoxing Li •

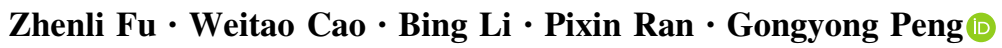

Published online: 18 September 2021

(C) Springer Nature B.V. 2021

Correction to: Cytotechnology (2021) 73(2):189-201 https://doi.org/10.1007/s10616-021-00456-5

This work was supported by the Local Innovative and Research Teams Project of Guangdong Pearl River Talents Program (2017BT01S155), the Natural Science Foundation of Guangdong Province (2021A1515011024, 2017A030313683, 2017A0303 10419), the National key Research and Development program of China (2016YFC1304100, 2016YFC130

The original article can be found online at https:// doi.org/10.1007/s10616-021-00456-5.

R. Liu - J. Xu - S. Li · Z. Fu · W. Cao .

P. Ran $(\bowtie) \cdot$ G. Peng $(\bowtie)$

Guangzhou Institute for Respiratory Health, State Key

Laboratory of Respiratory Disease, National Clinical

Research Center for Respiratory Disease, National Center

for Respiratory Medicine, The First Affiliated Hospital of Guangzhou Medical University, 151 Yanjiang Road,

Guangzhou 510120, China

e-mail: pxran@gzhmu.edu.cn

G. Peng

e-mail: gongyong19761@163.com

Y. Jiang

Respiratory Medicine, Hunan Provincial People's

Hospital, Changsha, China

W. Hong · B. Li

GMU-GIBH Joint School of Life Sciences, Guangzhou

Medical University, Guangzhou, China
4104, 2018YFC1311600), the National Natural Science Foundation of China (81570045), the Science and technology program of Guangzhou (201504010018), a Training Program for Academic Backbone of High Level Universities of Guangzhou Medical University (2017210) and a Grant of the First Affiliated Hospital of Guangzhou Medical University (201619). Dr. Peng was supported by the Scholarship of Guangzhou Medical University.

Publisher's Note Springer Nature remains neutral with regard to jurisdictional claims in published maps and institutional affiliations. 